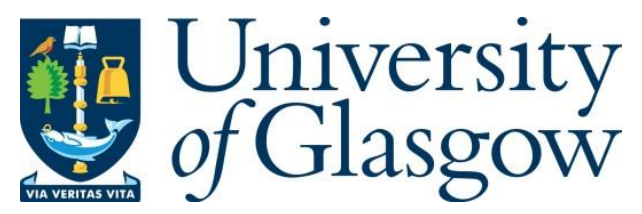

Gordon, N.A. et al. (2017) Experimental demonstration of coupled optical springs. Classical and Quantum Gravity, 34(3), 035020.

There may be differences between this version and the published version. You are advised to consult the publisher's version if you wish to cite from it.

http://eprints.gla.ac.uk/138051/

Deposited on: 13 March 2017

Enlighten - Research publications by members of the University of Glasgow http://eprints.gla.ac.uk 


\title{
Experimental demonstration of coupled optical springs
}

\author{
N A Gordon ${ }^{1}$, B W Barr ${ }^{1}$, A Bell ${ }^{1}$, C Graef $^{1}$, S Hild $^{1}$, \\ S H Huttner ${ }^{1}$, S S Leavey ${ }^{1}$, J Macarthur ${ }^{1}$, B Sorazu ${ }^{1}$, \\ J Wright ${ }^{1}$ and $\mathbf{K}$ A Strain ${ }^{1}$ \\ E-mail: neil.gordon@glasgow.ac.uk \\ ${ }^{1}$ SUPA, School of Physics and Astronomy, The University of Glasgow, Glasgow, \\ G12 8QQ, UK
}

\begin{abstract}
Optical rigidity will play an important role in improving the sensitivity of future generations of gravitational wave (GW) interferometers which employ high laser power in order to reach and exceed the standard quantum limit. Several experiments have demonstrated the combined effect of two optical springs on a single system for very low-weight mirror masses or membranes. In this paper we investigate the complex interactions between multiple optical springs and the surrounding apparatus in a system of comparable dynamics to a largescale GW detector. Using three $100 \mathrm{~g}$ mirrors to form a coupled cavity system capable of sustaining two or more optical springs, we demonstrate a number of different regimes of opto-mechanical rigidity and measurement techniques. Our measurements reveal couplings between each optical spring and the control loops that can affect both the achievable increase in sensitivity and the stability of the system. Hence this work establishes a better understanding of the realisation of the these techniques and paves the way to their application future GW observatories, such as upgrades to Advanced LIGO.
\end{abstract}

PACS numbers: $04.80 . \mathrm{Nn}, 95.75 . \mathrm{Kk}$

\section{Introduction}

The current network of gravitational wave detectors, comprises the two Advanced LIGO instruments [1], and GEO 600 [2] currently operating with Advanced Virgo [3] due to come on-line soon and KAGRA [4] due to start observing the next few years. These detectors are expected to be limited over most of their bandwidth by quantum noise. This noise arises from the statistical nature of light on detection and in its interaction with the suspended optics of the interferometer, resulting in the welldocumented Standard Quantum Limit [5] for a free-mass detector.

Optical springs have been shown to be capable of transforming the dynamics of the suspended optics in such an instrument into harmonic oscillators. By this means a narrowband increase in sensitivity could be created in the frequency band of the resulting resonance $[5,6,7,8]$. However, the sensitivity increase would be restricted to a narrow band around the resonance, and hence be of limited benefit. Further, a single optical spring is inherently unstable, requiring control systems to maintain 
stability of the opto-mechanical system $[9,10]$. Proposals exist to employ multiple optical springs in a single system, relatively tuned such that their combination is stable without requiring further complexity in the control loops [9]. In addition, they can be used to further re-shape the quantum sensitivity of the instrument, e.g. to produce enhancement over a wider band than is produced by a single resonance. In order that such a system may be realised in a large-scale interferometric gravitational wave detector, it is important that the practical aspects of its operation are well-understood.

Previous experiments by Corbitt et al [11] have demonstrated stable combination of two optical springs in a single opto-mechanical system on the $1 \mathrm{~g}$ scale. The investigations presented here are designed to demonstrate the feasibility of implementing such a system on a prototype $(\mathrm{kg})$ scale, with a view to inclusion in the design of future gravitational wave detectors.

We present the experimental apparatus and measurements performed at the Glasgow $10 \mathrm{~m}$ prototype interferometer laboratory to investigate the interactions between multiple optical springs and their control loops. The apparatus consists of two optical cavities coupled via a shared test mass. Each cavity is capable of sustaining an optical spring, the response of which is monitored by measurement of the open-loop transfer function of the feedback loops controlling each cavity length. Different regimes of opto-mechanical rigidity and measurement strategy are employed in order to examine the complex couplings that exist between the two optical spring effects and the control systems.

Specifically, the measurements presented in this paper aim to demonstrate that:

(i) the effective mass of the coupling optic is modified by the combined presence of two optical springs in accordance with the established opto-mechanical theory;

(ii) the frequency response of the opto-mechanical resonances coupling between cavities tends to be compensated by the loop gain, such that only an increased opto-mechanical stiffness is observed;

(iii) the coupled dynamics of a multiple optical spring system can be measured in the presence of control loops. We show that this can be achieved via a modification to the measurement technique, whereby the signal injections required for measurement of opto-mechanical effects are performed simultaneously in both cavities of the doubly-resonant system. This measurement technique can also be applied to demonstrate suppression of test-mass motion by the application of appropriately-tuned opto-mechanical stiffnesses, in the regime termed "annihilation" by Rehbein et al [9].

In Section 2 the theoretical foundations of opto-mechanical rigidity are introduced and the frequency-dependent optical spring constant, $K(\Omega)$, derived. In Section 3 the experimental concept and apparatus are described. In Section 4 we present simulations of the effect of two coupled optical springs on the control systems of a Fabry-Pérot cavity, and in Section 5 measurements performed in the doubly-resonant coupledcavity system to demonstrate and examine in detail points (i), (ii) and (iii) above. A summary of these measurements and outlook for future investigations are presented in Section 6. 


\section{Optical springs}

The optical spring regime in an optical resonator is achieved by detuning away from the point of resonance. For sufficiently small perturbations $x$ of the cavity length from the initial position of detuning (or equivalently for small perturbations of the frequency of the laser), the gradient of the intra-cavity power curve may be considered linear to first order. The optical spring then represents the predominantly linear coupling between cavity power and suspended optic position with restoring force, for small detuning relative to the cavity linewidth, expressed as $[12,13,8]$

$$
k_{O S}=\frac{8 \pi P_{0} F \theta}{\left.c \lambda\left(1+F \theta^{2}\right)^{2}\right)},
$$

where $P_{0}$ is the intra-cavity power, $\lambda$ is the laser wavelength, $F$ the coefficient of cavity finesse, $c$ the speed of light,

$$
F=\frac{4 \rho_{1} \rho_{2}}{\left(1-\rho_{1} \rho_{2}\right)^{2}},
$$

where $\rho_{1,2}$ are the amplitude reflectivities of the cavity optics, and $\theta=\frac{2 \pi x}{\lambda}$ describes the cavity detuning phase. The pendulum frequency is upshifted by the additional opto-mechanical stiffness to a frequency

$$
f_{O S}=\frac{1}{2 \pi} \sqrt{\frac{k_{O S}}{\mu}}
$$

where $\mu=\frac{m_{1} m_{2}}{m_{1}+m_{2}}$ is the reduced mass of a cavity bounded by masses $m_{1}$ and $m_{2}$.

The susceptibility is defined as the linear response function between force applied to and position of the test mass: $\chi=x(\Omega) / F(\Omega)$. In the presence of an optical spring this parameter can be expressed as

$$
\chi(\Omega)=\frac{1}{\mu\left(\Omega_{0}^{2}-\Omega^{2}\right)},
$$

where $\Omega$ is the frequency of observation and $\Omega_{0}=2 \pi f_{O S}$ is the upshifted pendulum frequency due to the additional stiffness of the optical spring. This parameter is characterised in the measurements of Section 5 to track the changing response of a test mass in the presence of two optical springs.

The simplified optical spring expression of Eq. 1 makes two key assumptions: that the mirrors are static, and that the fields circulating in the cavity respond instantaneously to any force. However, the optical power in the cavity responds to length changes on a time-scale consistent with the inverse of its linewidth, $\gamma$; when dynamic elements are introduced at frequencies of order $\gamma$, the frequency response of the system becomes important in deriving the true opto-mechanical response $[6,14,7]$. For detuning of order the cavity linewidth, the optical spring restoring force is modified to become

$$
K(\Omega)=k_{O S} \frac{1+\delta_{\gamma}}{\left(1+i \Omega_{\gamma}\right)^{2}+\delta_{\gamma}},
$$

where we have defined $\delta_{\gamma}=\delta / \gamma$ and $\Omega_{\gamma}=\Omega / \gamma, \delta$ is the detuning expressed as a fraction of the cavity linewidth and $\Omega$ is the observation frequency, and $k_{O S}$ is the static optical spring constant of Eq. 1. 
Noting the complex term, Eq. 5 can be separated into its real and imaginary parts:

$$
K_{O S}=K(\Omega)-i \Gamma(\Omega) .
$$

Since the second term is proportional to $i \Omega$, it can be thought of as a viscous damping term (alternatively as an "optical friction" [5]) which is always of opposite sign to the restoring force, $K$. Crucially, this indicates the optical rigidity is unstable. These instabilities are ordinarily corrected by the application of electronic feedback systems shaped in such a way as to stop the system from going unstable; however, with a second optical spring of appropriate detuning the system may be stabilised purely optically [9]. Configurations involving two or more optical springs can also allow reshaping of the noise spectral density of the system.

When two optical springs are combined in a cavity, their spring constants sum. If the desired effect is a stable opto-mechanical system, a high-power, highly-detuned positive spring, with a large restoring force and small anti-damping, can be combined with a lower-power less-detuned anti-spring, with small anti-restoring force and larger damping. The total optical rigidity is then arranged to have positive real and imaginary parts in Eq. 5 [15]:

$$
\begin{aligned}
K_{\text {total }}(\Omega) & =K_{O S, 1}(\Omega)+K_{O S, 2}(\Omega) \\
& =k_{O S, 1} \frac{1+\delta_{\gamma, 1}}{\left(1+i \Omega_{\gamma, 1}\right)^{2}+\delta_{\gamma, 1}}+k_{O S, 2} \frac{1+\delta_{\gamma, 2}}{\left(1+i \Omega_{\gamma, 2}\right)^{2}+\delta_{\gamma, 2}} \\
& =\left(K_{1}+K_{2}\right)-i\left(\Gamma_{1}+\Gamma_{2}\right) .
\end{aligned}
$$

By combining optical springs with appropriate detuning and laser power, then, the combined effect can be very beneficial to the operation or sensitivity of an interferometric gravitational wave detector; stability can be improved resulting in reduced complexity in the control systems, and the quantum-limited sensitivity improved over a wider band than would be possible for the single optical spring case.

\section{Experimental setup}

The experimental system consists of two Fabry-Perot cavities coupled mechanically via a shared test-mass. The opto-mechanical properties of interest can be observed and characterised by performing transfer function measurements in the feedback loops required to maintain the length of the cavities. In this section we describe the implementation of the apparatus within the existing infrastructure of the Glasgow $10 \mathrm{~m}$ prototype interferometer lab, as well as simulation and measurement of the open-loop transfer functions of each cavity control loop in which opto-mechanical effects will be observed.

A schematic of the system is shown in Figure 1. Three suspended $100 \mathrm{~g}$ optics, the Left, Right and Central Test Masses (LTM, RTM and CTM), define the two mechanicallycoupled cavities. The most efficient way to fit these two cavities into the available vacuum space was determined to be folding of each cavity, requiring the addition of an extra optic in each - the Left and Right Steering Mirrors (LSM and RSM) - at $45^{\circ}$ to the circulating fields. These folding optics contribute optical losses of comparable magnitude to the main cavity optics, resulting in a moderately lower finesse in each cavity than would be expected. The pertinent parameters following characterisation 


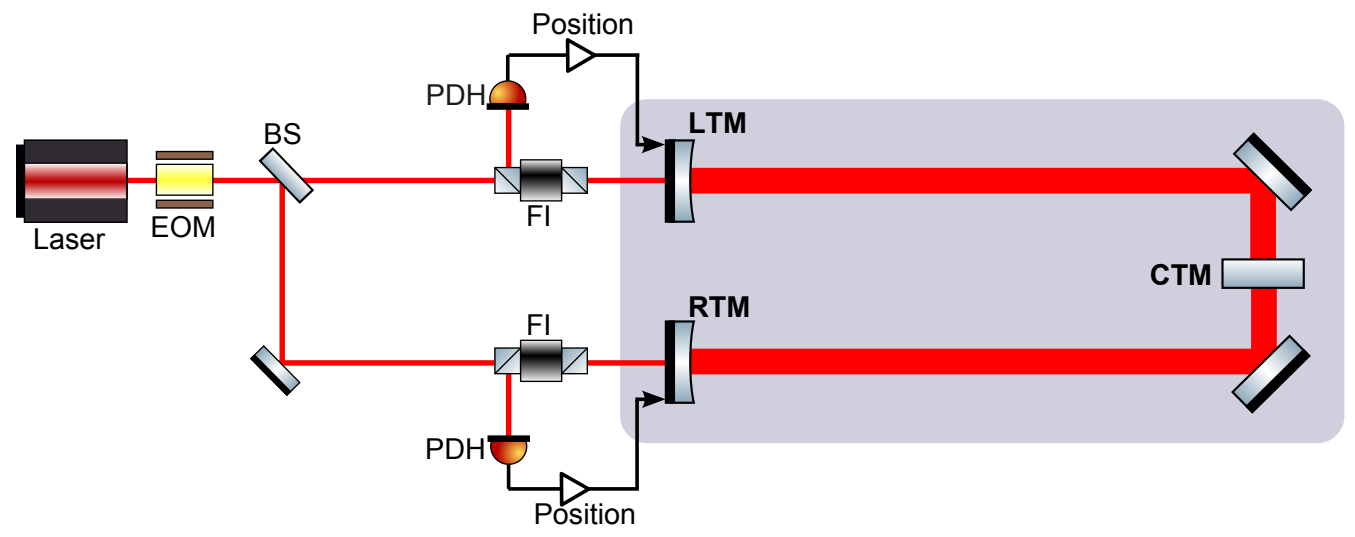

Figure 1: Simplified schematic diagram of the experimental setup. A single $1064 \mathrm{~nm}$ source laser illuminates two $10 \mathrm{~m}$ optical cavities, folded into a single $10 \mathrm{~m}$ length of vacuum tube. The two cavities are formed by the Left Test Mass (LTM), Right Test Mass (RTM) and coupling Central Test Mass (CTM), with cavity-folding optics termed the Left and Right Steering Mirror (LSM and RSM). Pound-Drever-Hall (PDH) error signals are obtained from the reflected optical signal from each cavity, accessed via a Faraday isolator (FI) in each path, and used to apply a feedback signal for precision control of each cavity length.

\begin{tabular}{c|c|l} 
Parameter & Value & Description \\
\hline$M_{L T M}, M_{R T M}$ & $99.3 \mathrm{~g}$ & LTM and RTM test masses \\
$M_{C T M}$ & $102 \mathrm{~g}$ & CTM test mass \\
$\tau_{L T M}^{2}$ & $100 \mathrm{ppm}$ & LTM power transmission \\
$\tau_{R T M}^{2}$ & $100 \mathrm{ppm}$ & RTM power transmission \\
$R_{L T M}$ & $15 \mathrm{~m}$ & LTM radius of curvature \\
$R_{R T M}$ & $15 \mathrm{~m}$ & RTM radius of curvature \\
$\mathcal{F}_{L}$ & 10220 & Left-hand cavity finesse \\
$\mathcal{F}_{R}$ & 9155 & Right-hand cavity finesse \\
$\gamma_{L}$ & $734 \mathrm{~Hz}$ & Left-hand cavity linewidth \\
$\gamma_{R}$ & $820 \mathrm{~Hz}$ & Right-hand cavity linewidth \\
$P_{i n}^{L}$ & $1.65 \mathrm{~W}$ & Left-hand cavity input power \\
$P_{i n}^{R}$ & $1.85 \mathrm{~W}$ & Right-hand cavity input power
\end{tabular}

Table 1: System parameters.

of the system are presented in Table 1.

The experimental setup allows optomechanical rigidity to be induced in either or both cavities by correct tuning of the LTM and RTM positions. Digital control systems were implemented using the Control and Data System (CDS) [16], developed primarily for use at the LIGO sites but also adopted at Glasgow. Figure 2 shows a simplified block diagram of the control loop used for one cavity to control the position of the input test mass. The transfer function measurements presented in this paper are performed by injecting appropriate signals into the control loops and reading out the loop gain response via the CDS. 


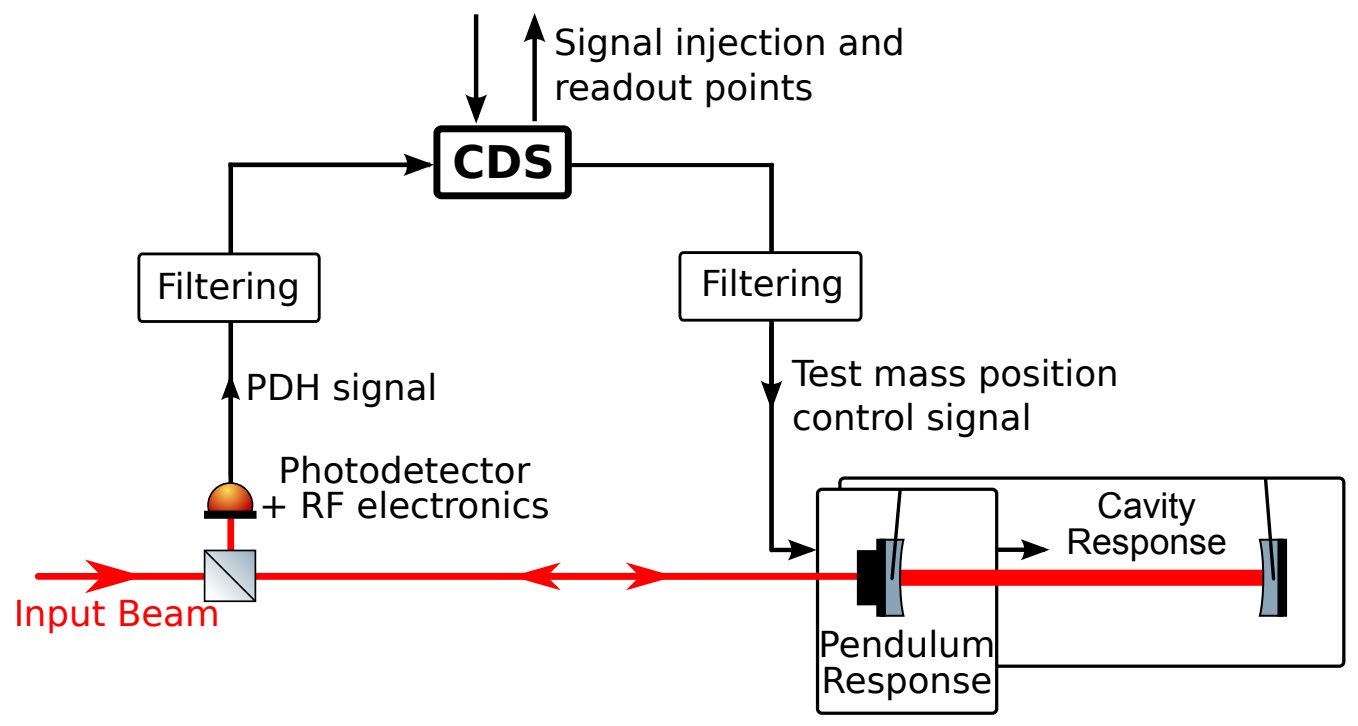

Figure 2: Simple block diagram of the control loop for each cavity. Pound-Drever-Hall control signals are appropriately filtered to construct the feedback signal for precision control of the position of each input test mass. Injection and readout signals are applied via the $C D S$.

The experiments detailed in this paper are designed to monitor optical spring resonant features from approximately $100 \mathrm{~Hz}$ up to around $1 \mathrm{kHz}$. In order to reduce the frequency noise on the light over this range, it was necessary to pre-stabilise the source laser by locking it to a separate $10 \mathrm{~m}$ suspended-optics reference cavity, achieved by active feedback. The two coupled optical spring cavities are then locked to the laser frequency via feedback to the positions of the input test masses to reduce the residual error to below a picometre. This feedback is controlled via servos implemented in the CDS, which allows flexible filtering and signal injection capabilities when compared to the analogue systems used in the past. Since the measurement region extends up to a kilohertz in frequency, for appropriate stability of the control loops the servos are designed to give unity gain points of at least $1 \mathrm{kHz}$.

Monitoring of the reflected signals from each cavity reveals fundamental mode fringes of duration $\sim 100 \mu \mathrm{s}$ as each cavity swings freely through resonance. The CDS imposes an inherent phase delay upon any propagating control signals that makes acquisition of lock during these short fringe time-scales, as well as stable lock with unity gain frequencies $\geq 1 \mathrm{kHz}$, very challenging. To overcome this obstacle, a novel modification to the Pound-Drever-Hall control signals to each cavity was implemented. In this method, the normal PDH signal is divided by a signal proportional to the power in each cavity. This has the effect of significantly widening the capture range of the feedback loops, with the added benefit of allowing each cavity to be detuned significantly wider than would ordinarily be possible with a regular PDH feedback signal. This method has been developed from one previously described by M. Evans [17]. Its implementation and characterisation at the Glasgow $10 \mathrm{~m}$ prototype laboratory is the 

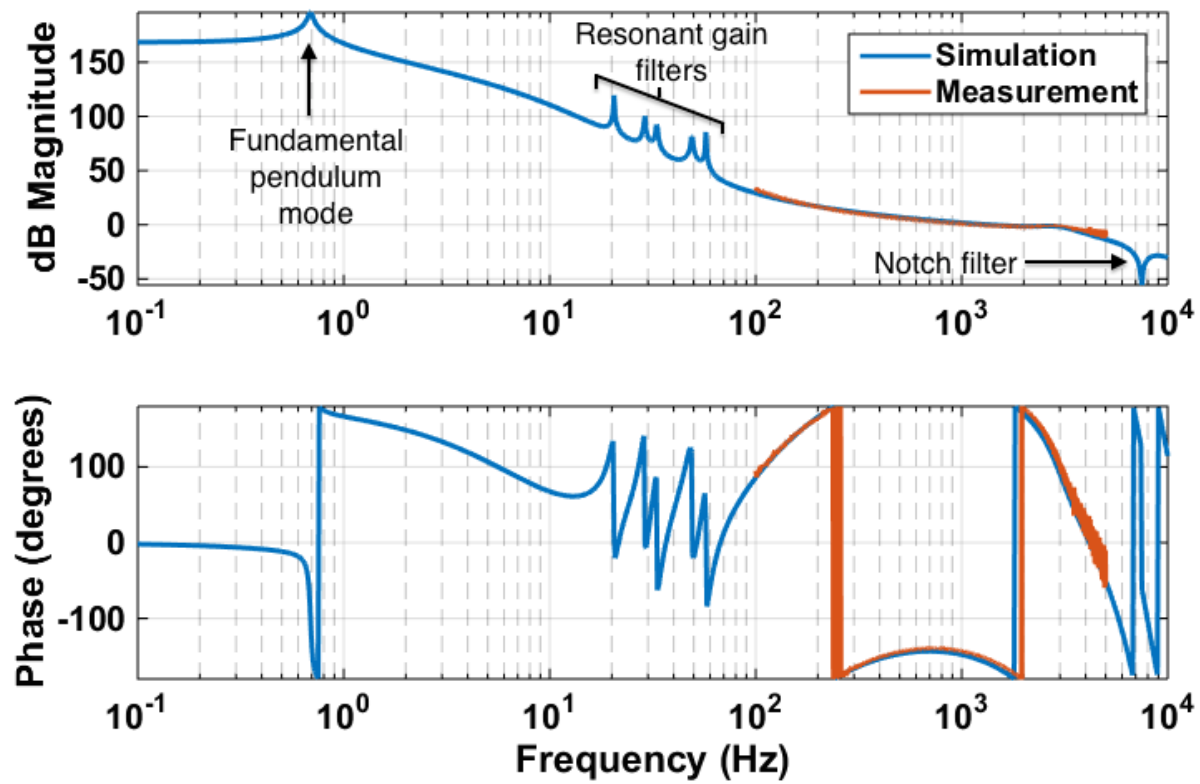

Figure 3: Simulation and measurement of the complete open-loop transfer function for one cavity, indicating unity gain point at approximately $1.3 \mathrm{kHz}$. The simulated OLTF is observed to fit the measurement to within $\pm 2 \mathrm{~dB}$ and $\pm 7^{\circ}$ through the region $100 \mathrm{~Hz}$ to $3 \mathrm{kHz}$. The resonance at $0.69 \mathrm{~Hz}$ is the fundamental longitudinal pendulum mode; the five resonant features in the $20-60 \mathrm{~Hz}$ range are resonant gain filters, designed to reduce excitation of prominent suspension modes at these frequencies; the notch at $7.5 \mathrm{kHz}$ exists to mitigate excitation of a (presumed) internal mode of the test mass at this frequency.

subject of a paper under preparation [18].

Figure 3 compares a simulation and measurement of the complete open-loop transfer function (OLTF) for one of the two optical spring cavities, indicating a unity-gain point of $\sim 1.3 \mathrm{kHz}$. The measured OLTF is shown to very closely match the simulation, indicating that the elements of the control loops are well-understood. The two cavities are of nominally identical design, such that the measured OLTF is representative of either. To avoid excitation of mechanical modes of the test mass suspensions that would result in loss of lock, measurements of the open-loop gain to monitor optomechanical resonant features can only easily be performed down to approximately $100 \mathrm{~Hz}$.

\section{Simulations}

Prior to performing measurements, simulations of the anticipated open-loop resonant response of each cavity were performed in Optickle [19], a set of scripts designed to be run within MATLAB ${ }^{\circledR}[20]$ which together may be used to build frequency-domain models of interferometers or similar optical systems. 

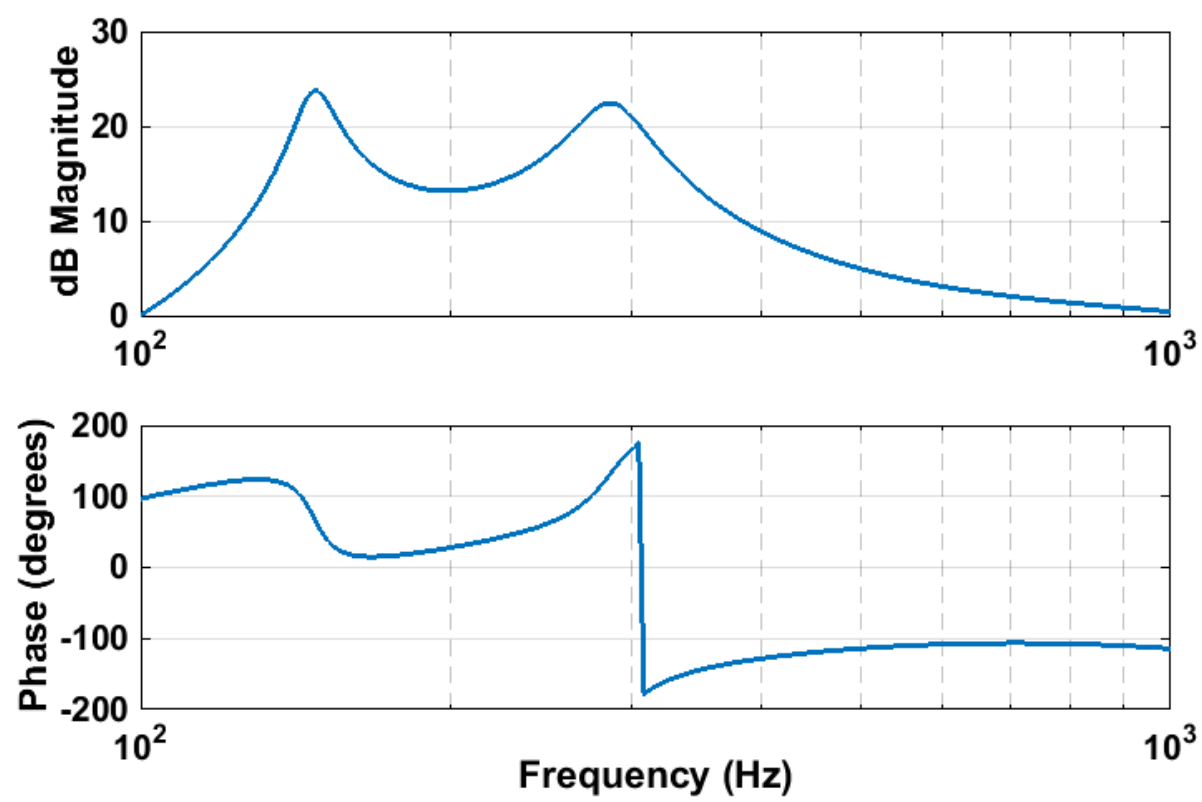

Figure 4: Coupled spring simulation including all the elements of the control loop contributing to Figure 3. This simulated transfer function is performed inside the right-hand cavity control loops, with detunings of $\delta_{L}=0.3 \gamma_{L}$ and $\delta_{R}=1 \gamma_{R}$. Note the pair of coupled opto-mechanical resonances at approximately $150 \mathrm{~Hz}$ and $285 \mathrm{~Hz}$.

Figure 4 displays the expected open-loop transfer function evaluated in one cavity, for the case where an optical spring is present in both cavities. All of the filters that constitute the open-loop response of the control loop, shown in Figure 3, have been included in the simulation. The optical and mechanical parameters are the same as those listed in Table 1. This simulation represents one possible modification to the onresonance open-loop transfer function shown in red in Figure 3. Two opto-mechanical resonant features are evident in the cavity which is simulated, with the purely optical resonance occurring at a frequency above the measurement band. These show up as a result of their common interaction with the Central Test Mass. The stiffness of each cavity has been modified with respect to the single-spring case in accordance with Eqs. 3 and 7, and hence the resonant features are altered. The resulting modification of the response of the suspended CTM is then imposed upon the open-loop transfer function of the cavity under observation. The predictions of these simulations are compared to measurements performed in individual cavities of the doubly-resonant system in Section 5.2.

\section{Measurements}

In the frequency range of interest for our experiment, laser frequency noise is dominant over other noise contributions such as thermal noise on the optic coatings and seismic 
noise of the surroundings, each of which is of higher magnitude than the quantum noise on the light that is expected to be improved or modified by the presence of optical rigidity. For this reason, in this system, the effect of opto-mechanical rigidity cannot be evaluated by monitoring the total noise budget; instead, our measurement plan identifies and characterises opto-mechanical effects via their influence upon the control loops for each cavity, by monitoring of the open-loop transfer function of each cavity.

Broadband white noise may be injected on to the input test mass positions via the CDS (as shown in Figure 2) and the subsequent response of the PDH error signal monitored in order to obtain the open-loop transfer functions of each feedback loop, from which opto-mechanical effects are inferred.

In the first set of measurements, presented in Section 5.1, the control loop and optical models introduced in Sections 3 and 4 are verified by comparison of simulated and measured cavity OLTFs when a single optical spring is present.

In Section 5.2, we demonstrate that the effective mass of the coupling optic is modified by the presence of two optical springs acting in combination in accordance with the established opto-mechanical theory. This is manifest as a modification to the singlespring mechanical response when opto-mechanical rigidity is present in both cavities, consistent with Eq. 7. It is noted that since the anticipated coupled resonant responses lie beneath the unity gain points of the control loops of both cavities, these features are compensated by the loop gain.

In a third set of measurements, presented in Section 5.3, a modification to the measurement technique is introduced: transfer functions are performed simultaneously in the control loops of both cavities in the doubly-resonant system. This makes visible the coupled resonant responses that would otherwise be compensated by the loop gain, as well as more complex features resulting from various couplings of the optical springs and measurement signals to the loop gains.

Finally, in Section 5.4 optical "trapping" or "annihilation" is demonstrated, whereby approximately equal opto-mechanical effects are generated on either side of the coupling optic to reduce its motion.

\subsection{Verification of Models}

To confirm that the control system and optical models presented in Sections 3 and 4 are valid, open-loop transfer function measurements were performed in a single detuned cavity and compared with modelling predictions. Examples of this, depicting the effect of individual optical springs with increasing detuning upon the OLTF of a single cavity, are shown in Figure 5. The modelled responses are observed to fit to the measurements with $R^{2}>0.98$, and so we conclude that our models are realistic. These examples are representative of measurements performed in both cavities of the system. 

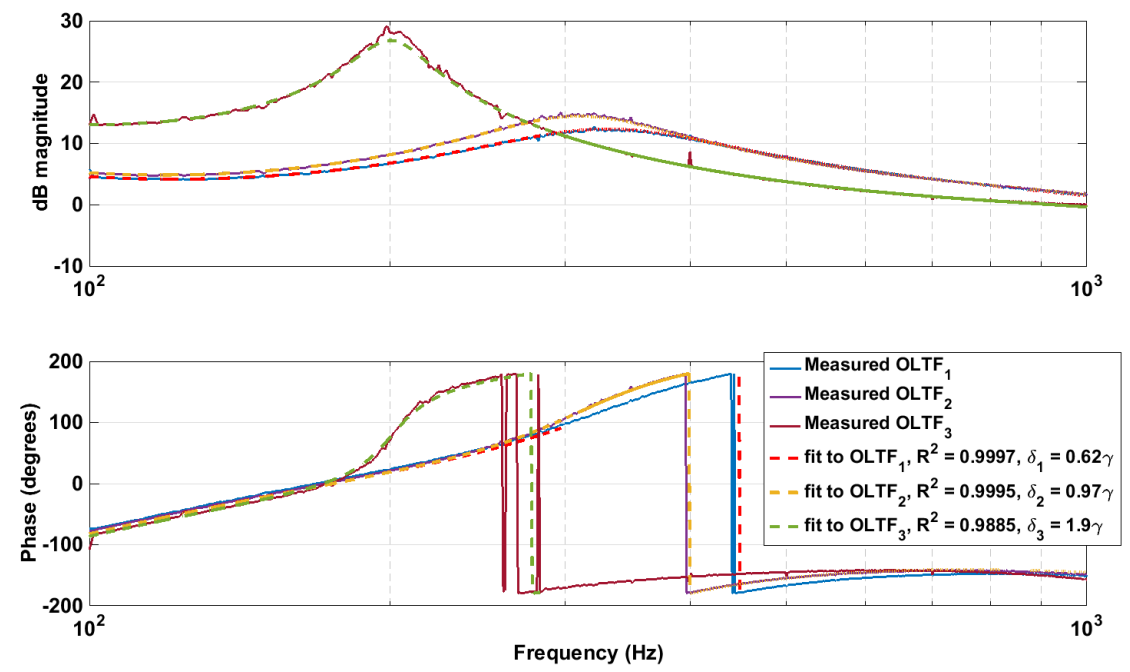

Figure 5: Magnitude and phase of open-loop transfer functions (measured between the injection and readout points indicated in Figure 2) showing examples of optomechanical resonances for three different values of detuning in the right-hand cavity. The fitted models were simulated in Optickle, as described in Section 4, and are observed to accurately recreate the measured responses.

\subsection{Modification of Single-Spring Dynamics}

Here we demonstrate that the stiffness and hence resulting resonant frequency and effective mass of the CTM suspension are modified by the presence of opto-mechanical effects on both sides. We consider the case where an optical spring at a particular detuning is monitored inside the control loops of one cavity while the detuning of the adjacent cavity is adjusted. Modifications to the measured opto-mechanical resonance are then shown to be consistent with the theory.

Figure 6 shows OLTF measurements of the right-hand cavity, measured between the injection and readout points in CDS depicted in Figure 2. Throughout the measurements shown, this cavity maintains an optical spring with detuning of $0.7 \gamma_{R}$; this value is chosen to yield a resonance that is relatively strong, but weak enough that modifications due to the second opto-mechanical coupling are visible. For each plotted curve, the detuning applied to the adjacent left-hand cavity is adjusted, such that the second opto-mechanical coupling increases in stiffness to approach the maximum strength coupling at $0.57 \gamma_{L}$.

The gain of the resonance in the right-hand cavity is observed to increase by $2.5 \mathrm{~dB}$, or a factor of 1.33 , between the point at which there is no opto-mechanical rigidity in the adjacent left-hand cavity $\left(\delta_{L}=0\right.$ in Figure 6$)$ and the point at which it is at maximum strength $\left(\delta_{L}=0.539 \gamma_{L}\right)$. Since by the latter point the CTM has become rigidly coupled to the LTM, its effective mass as observed within the right-hand cavity frame has doubled. This means that the reduced mass of the right-hand cavity 


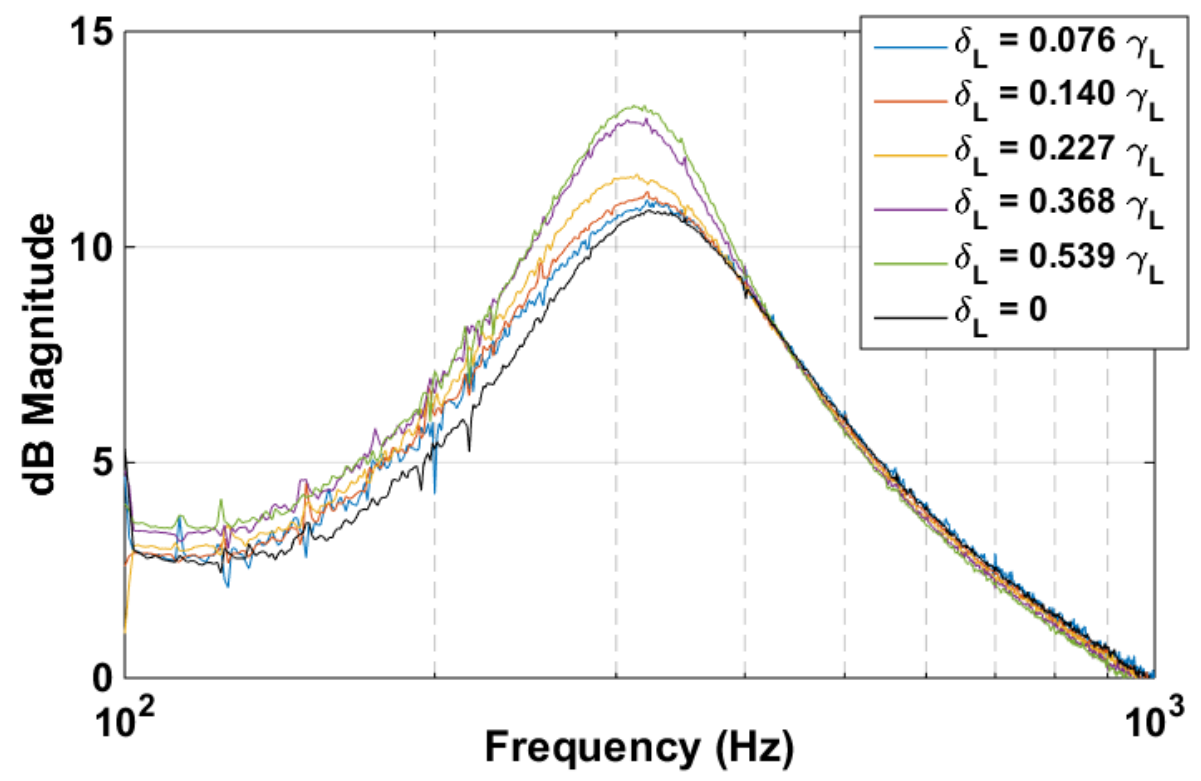

Figure 6: Open-loop transfer function measurements of the right-hand cavity. The right-hand cavity is fixed at a detuning of $\delta_{R}=0.7 \gamma_{R}$ while the left is detuned from $\delta_{L}=0$ to $\delta_{L}=0.539 \gamma_{L}$. The magnitude of the observed opto-mechanical resonance in the right-hand cavity is observed to be modified by the rigidity of the left, in good agreement with the predictions of opto-mechanical theory. This is examined in detail in the text.

has increased from $0.05 \mathrm{~kg}$ (when $M_{R T M}=M_{C T M} \simeq 100 \mathrm{~g}$ ) up to $0.067 \mathrm{~kg}$ when the CTM and LTM are coupled $\left(M_{C T M} \simeq 200 \mathrm{~g}\right)$; according to Eq. 4 , this results in a change in susceptibility of the right-hand cavity of $\Delta \chi=1.33$. This prediction is in good agreement with the observed resonant magnitude change, and thus we infer that the observed coupling of opto-mechanical springs in Figure 6 is consistent with the predictions of the established theory.

Note that only a single resonance is observed in this measurement, in contrast with the modelled doubly-resonant system presented in Figure 4 which indicates that two coupled resonances ought to be present when the cavities are mechanically coupled and both detuned. However, model and measurement are reconciled when the closed-loop feedback of each cavity is considered - this is not present in the Optickle models, which consider only the circulating fields and mechanical transfer functions of the system. In the presence of the control loops, each cavity is acted upon via the input test mass to counteract any excess relative motion that would modulate the total length. The result of this is that outside each control loop the opto-mechanical resonance is not observed, because a control signal of appropriate size is being fed-back to compensate the effect; instead, only the extra stiffness arising from the coupling of each input test mass to the CTM is observed in the adjacent cavity, manifesting as the changes to the susceptibility of the coupled-spring system observed in Figure 6. 
These measurements confirm that opto-mechanical rigidities couple as expected from the associated changes to the susceptibility of each cavity. Since the measurements are performed beneath the unity gain point of each system, coupled resonant features are observed to be compensated by the loop gain. This is an important observation as regards the "optical bar" proposal for an interferometric gravitational wave detector, whereby an optical spring in each arm couples to either side of a shared mass, with opto-mechanical resonant frequencies likely below the unity gain points of the control systems.

\subsection{Measurement of Coupled Opto-Mechanical Dynamics}

Section 5.2 demonstrates that the coupled resonant features of a multiple optical spring system are suppressed in the presence of the control loops. Accordingly, a means of measurement has been developed in which these coupled resonant features are made visible. This is based upon simultaneous injection of an identical signal into both cavities and simultaneous measurement of both open-loop transfer functions. Since a coherent signal is now observed in both cavities at the CTM, shaped by the gain in each feedback loop, the closed-loop dynamics of one cavity (including any optomechanical resonant features) can couple into the other. This is due solely to optical forces acting upon the CTM, applied via the opto-mechanical coupling to each ITM, which is then read out coherently in the measurement of each cavity.

In the first demonstration of this technique, one cavity is held at the resonance point $\left(\delta_{L}=0\right)$ and the other is detuned $\left(\delta_{R} \neq 0\right)$. An identical excitation signal is applied to both ITM positions, and the open-loop response of both cavities monitored simultaneously. Measurement of each cavity separately would result in observation of a well-defined opto-mechanical resonance in the detuned cavity and of the on-resonance response (similar to Figure 3) in the other. Using this new measurement technique, we find that the on-resonance cavity also displays resonant features consistent with opto-mechanical coupling.

The result is shown in Figure 7. The black curve depicts the left-hand cavity OLTF when both cavities are held on-resonance; the red and blue curves correspond to OLTF measurements of the right- and left-hand cavities respectively after an optical spring with detuning $\delta_{R}=1 \gamma_{L}$ is introduced into the right-hand cavity. This detuning is chosen to yield a relatively high- $Q$ spring resonance in the middle of the measurement region. The left-hand cavity is held on-resonance $\left(\delta_{L}=0\right)$ throughout. A strong opto-mechanical coupling is observed in the $300 \mathrm{~Hz}$ region in both cavities. This demonstrates measurement of coupled opto-mechanical dynamics in the presence of cavity control loops.

The observation shown in Figure 7 is enabled by the simultaneous measurement of the two cavities. Below the associated resonant frequency of the detuned cavity, the CTM is coupled rigidly to the input mirror. This means that the excitation signal moves the CTM dynamically according to the power gain in that cavity, which is now subject to a broad resonance at the spring frequency. The adjacent on-resonance cavity then reads out this excess motion of the CTM coherently, resulting in a modification to the expected $\delta_{\gamma}=0$ transfer function. 

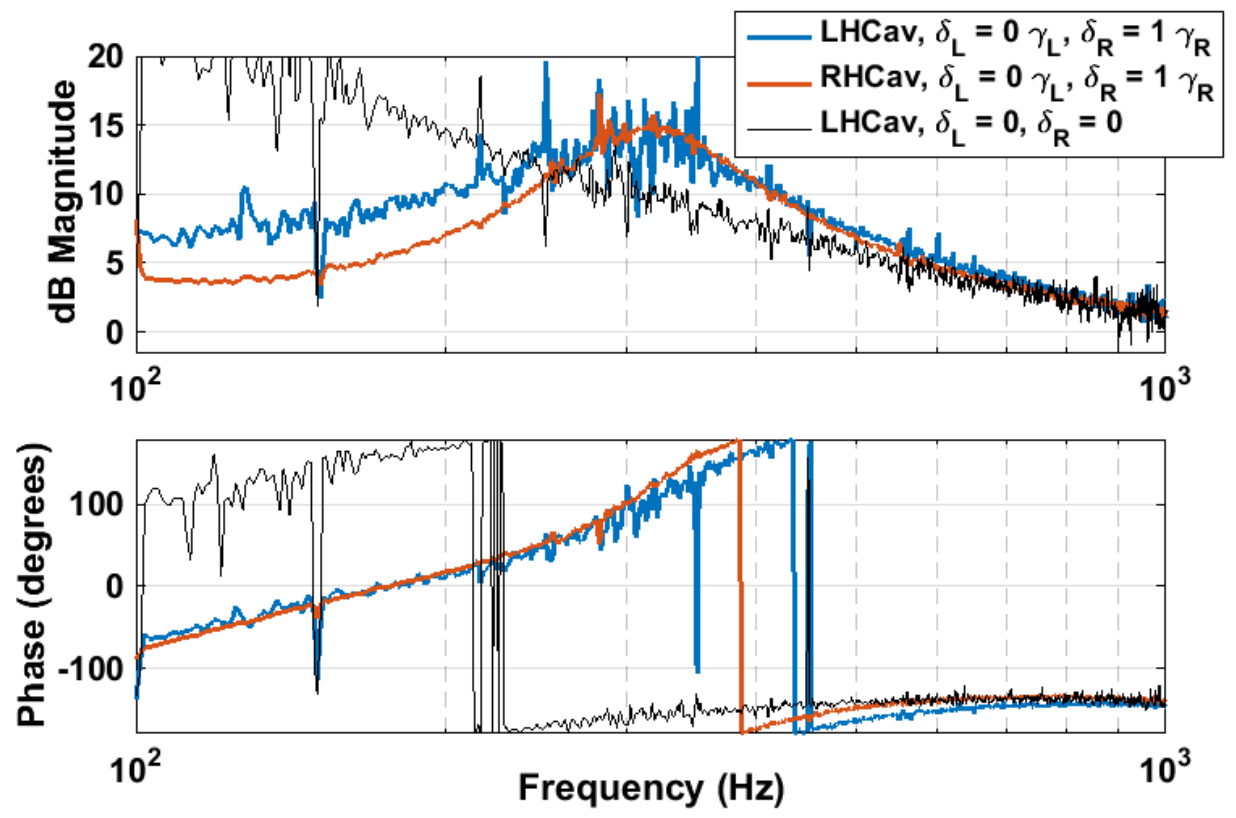

Figure 7: Observation of an optical spring in the right-hand cavity coupling into the open-loop transfer function of the on-resonance left-hand cavity. The black plot indicates the OLTF of the left-hand cavity when both are held on-resonance. The red and blue plots show the OLTFs of the right- and left-hand cavities respectively when the right-hand cavity is detuned to $\delta_{R}=1 \gamma_{R}$. An opto-mechanical resonance is observed in both cavities in the region of $300 \mathrm{~Hz}$.

The method of simultaneous measurement can be further applied to observe instabilities in the closed-loop feedback of one cavity coupling into the dynamic response of the other. This is shown in Figure 8. In this instance, the right-hand cavity is detuned to $\delta_{R}=1 \gamma_{R}$; this is again chosen to yield a relatively high- $Q$ resonance in the middle of the measurement range at approximately $300 \mathrm{~Hz}$. As before, this spring feature is observed to couple from the right-hand cavity (red curve) to the left-hand cavity (blue curve). A detuning of $\delta_{L}=-0.58 \gamma_{L}$ is also applied to the left-hand cavity, chosen to give a near-maximum strength anti-spring in that cavity, with the result that the open-loop gain of the left-hand cavity is observed to approach unity at $180 \mathrm{~Hz}$. The unity gain instability will result in a damped oscillation in the closed-loop left-hand cavity gain at $180 \mathrm{~Hz}$. This modulates the position of the CTM, such that the lefthand cavity gain-instability is visible in the right-hand cavity OLTF. Note that the right-hand cavity response is very similar to the model presented in Figure 4, in which two positive springs are simulated with coupling via the CTM.

The potential instability can be mitigated by application of electronic gain in the left-hand cavity in which the anti-spring is present. This may be required in a system such as that proposed by Rehbein et al. [9] in which an anti-spring is combined with 

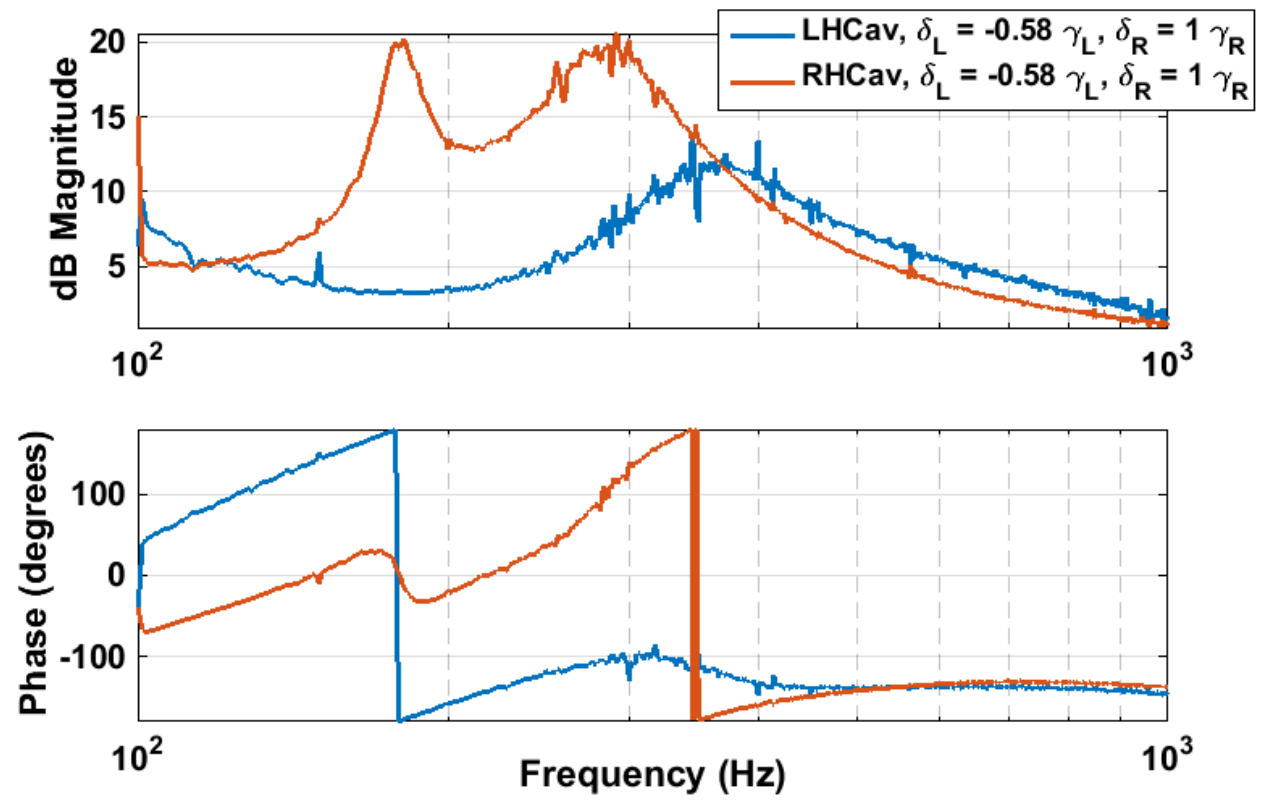

Figure 8: Simultaneous measurements showing a resonant feature resulting from a unity-gain instability at approximately $180 \mathrm{~Hz}$ in the closed-loop response of the lefthand cavity (blue) coupling into the open-loop gain of the right-hand cavity (red). The coupled resonant feature and optical spring in the right-hand cavity OLTF agree well with the double-spring simulation presented in Figure 4.

a positive spring in order to form a stable net opto-mechanical effect, or similarly in the optical bar interferometer configuration [21].

\subsection{Optical Trapping}

The final observation made is that, when there is a coherent signal injected onto both ITMs which couples to the CTM through optical rigidity in both cavities, the apparent motion of the CTM is suppressed. This behaviour is evident in the measurements presented in Figure 9.

When compared with the equivalent single-spring measurements (thin red and blue curves in Figure 9), for which the adjacent cavity is not detuned, we note a reduction in low-frequency gain when positive optical springs are introduced into both cavities. This effect is more significant in the left-hand cavity (thick blue), as its detuning has been chosen to be much larger than that of the right-hand cavity to yield lower optical power and weaker opto-mechanical coupling. Consequently, due to stronger opto-mechanical coupling and higher circulating power, the suppressed response in the right-hand cavity (thick red) is less obvious. This open-loop gain reduction is indicative of reduced CTM motion over these frequencies. In particular, the OLTF of the left-hand cavity in the double-spring measurement is observed to fall below unity below $200 \mathrm{~Hz}$. Were this a physical effect upon the loop gain, this ought to result in 

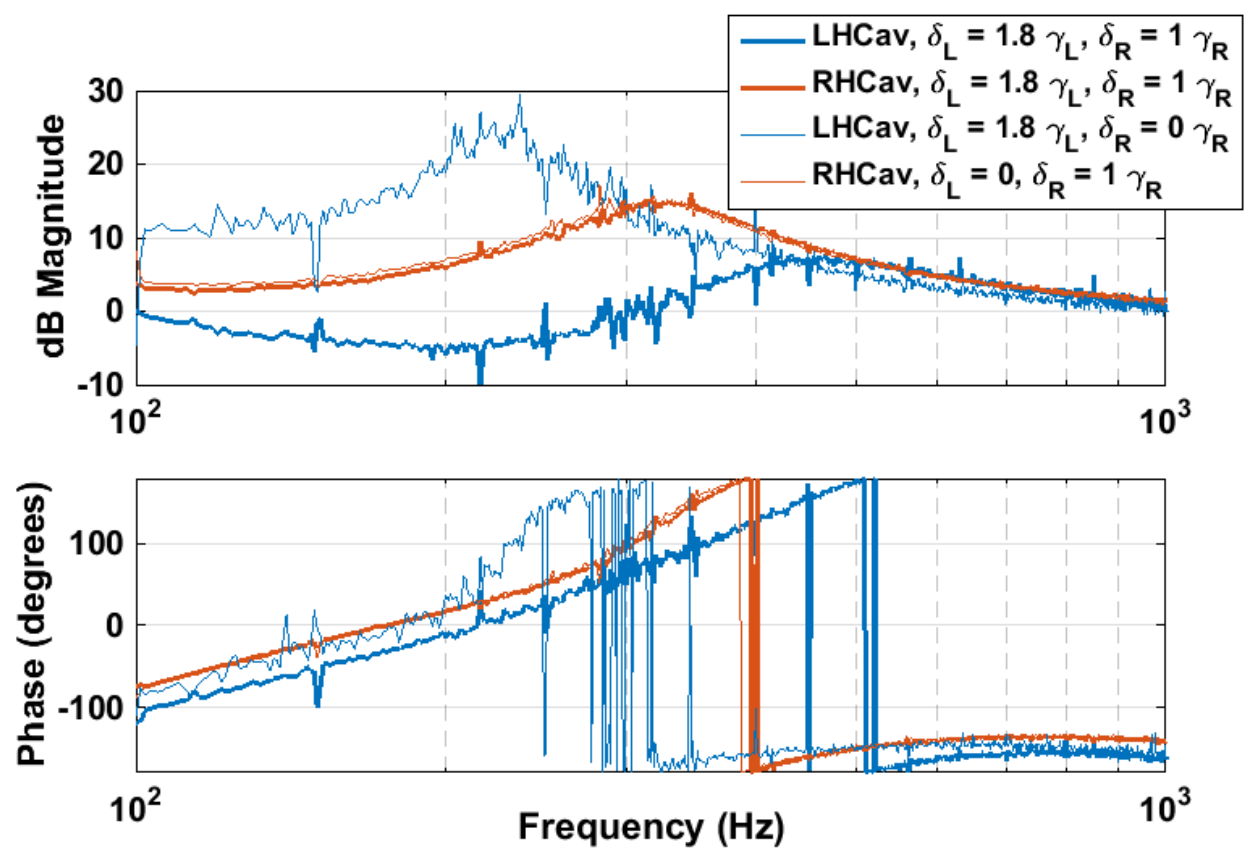

Figure 9: Simultaneously-performed open-loop transfer functions showing apparent suppression of CTM motion as observed by both cavities (thick lines) when compared to the equivalent single-spring OLTFs (thin lines). The left-hand cavity (blue) shows significant reduction in open-loop gain when the motion of the CTM is constrained by the action of both optical springs on either side.

a unity gain instability at this frequency; this backs up the notion that this effect is imposed upon the measurement only due to the apparent reduced motion of the CTM, rather than on the loop gain itself. Note the similarity between this measurement and the "annihilation" double-spring regime proposed by Rehbein et al. [9], whereby the two springs are tuned to "trap" the CTM longitudinally by application of equal optical force on either side.

\section{Summary and Future Work}

We have presented the design of a facility for the investigation of coupled optical springs at the Glasgow $10 \mathrm{~m}$ Prototype Interferometer Laboratory. Using this apparatus, several different regimes of coupled opto-mechanical rigidity, relevant to potential future applications of optical rigidity in gravitational wave detectors have been demonstrated.

Coupled optical springs were observed to behave as expected in the dual-cavity system. The dynamics of the coupling optic were modified in a manner consistent with the theory. Importantly, we confirm that resonant features from one cavity are not observed to couple into the open-loop measurements of the other cavity, as they are compensated for by the control loop gain. This result applies in particular to the design of 
'optical bar' detectors, whereby the end mass of each interferometer arm is coupled to a central mirror with optical springs; the opto-mechanical rigidity in each arm will be modified via the interaction at the shared optic, and it is likely that the optical springs will be situated beneath the unity gain point of the feedback loops for stability.

To make coupled resonant features visible, a method of simultaneous signal injections into both cavities was developed and applied. It was shown that using this scheme the resonant response of the optical spring in one cavity is observed to couple into the onresonance response of the adjacent cavity. A second measurement indicated that unity gain instabilities in one cavity can couple into the open-loop gain of the adjacent cavity. Each of these effects is interpreted as resulting from the simultaneous injection of coherent signals upon the CTM from either cavity. These effects will be important for any multiple-spring system in which a coherent signal influences coupled control loops.

Finally, with an optical spring in both cavities the simultaneous injection was observed to result in radiation pressure couplings to both sides of the CTM, effectively suppressing its motion.

\section{Acknowledgments}

This work was supported by the University of Glasgow and by the Science and Technology Facilities Council: grant numbers ST/I001085/1 and ST/L000946/1.

\section{References}

[1] B. P. Abbott, R. Abbott, R. Adhikari, et al., "LIGO: the Laser Interferometer Gravitational-Wave Observatory," Reports on Progress in Physics, vol. 72, no. 7, 076901, p. 076 901, Jul. 2009. arXiv: 0711.3041 [gr-qc].

[2] H. Grote, "The geo600 status," Class. Quantum Grav., vol. 27, p. 084 003, 2010.

[3] F. Acernese, M. Agathos, K. Agatsuma, et al., "Advanced Virgo: a secondgeneration interferometric gravitational wave detector," Classical and Quantum Gravity, vol. 32, no. 2, 024001, p. 024 001, Jan. 2015. arXiv: 1408.3978 [gr-qc].

[4] K. Somiya, "Detector configuration of KAGRA-the Japanese cryogenic gravitational-wave detector," Classical and Quantum Gravity, vol. 29, no. 12, 124007, p. 124007, Jun. 2012. arXiv: 1111.7185 [gr-qc].

[5] S. L. Danilishin and F. Y. Khalili, "Quantum measurement theory in gravitational wave detectors," Living Reviews in Relativity, 2012.

[6] F. Y. Khalili, "Frequency-dependent rigidity in large-scale interferometric gravitational-wave detectors," Physics Letters A, vol. 288, pp. 251-256, Oct. 2001. eprint: gr-qc/0107084.

[7] T. Corbitt, D. Ottaway, E. Innerhofer, et al., "Measurement of radiationpressure-induced optomechanical dynamics in a suspended fabry-perot cavity," Physical Review A (Atomic, Molecular, and Optical Physics), vol. 74, no. 2, 021802, p. $021802,2006$.

[8] M. Rakhmanov, "Dynamics of laser interferometric gravitational wave detectors," PhD thesis, California Institute of Technology, 2000. 
[9] H. Rehbein, H. Müller-Ebhardt, K. Somiya, et al., "Double optical spring enhancement for gravitational-wave detectors," Phys. Rev. D, vol. 78, no. 6, pp. 062 003-+, Sep. 2008. arXiv: 0805.3096.

[10] V. Chickarmane, S. V. Dhurandhar, R. Barillet, et al., "Radiation Pressure and Stability of Interferometric Gravitational-Wave Detectors," Applied Optics, vol. 37, pp. 3236-3245, May 1998.

[11] T. Corbitt, Y. Chen, E. Innerhofer, et al., "An all-optical trap for a gram-scale mirror," Phys. Rev. Lett., vol. 98, no. 15, p. 150802 , Apr. 2007.

[12] M. P. Edgar, "Experimental investigations into diffractive optics and optomechanical systems for future gravitational wave detectors," $\mathrm{PhD}$ thesis, University of Glasgow, 2011.

[13] J. Macarthur, "Towards surpassing the standard quantum limit using optical springs," PhD thesis, University of Glasgow, 2014.

[14] V. B. Braginsky and S. P. Vyatchanin, "Low quantum noise tranquilizer for Fabry-Perot interferometer," Physics Letters A, vol. 293, pp. 228-234, Feb. 2002.

[15] H. Miao, Exploring Macroscopic Quantum Mechanics in Optomechanical Devices. 2012.

[16] R. Bork, "AdvLigo CDS Design Overview," LIGO Scientific Collaboration, Tech. Rep., 2010.

[17] M. Evans, "Lock Acquisition in Resonant Optical Interferometers," PhD thesis, California Institute of Technology, 2002.

[18] B. W. Barr, A. Bell, N. A. Gordon, et al., "Widened signals for the control and manipularion of detuned optical resonators," In preparation, 2016.

[19] M. Evans, "Optickle," Technical Report T07026-00, LIGO Laboratory, Tech. Rep., 2007.

[20] MathWorks Inc., MATLAB release R2014b. Natick, Massachusetts: MathWorks Inc., 2014.

[21] V. B. Braginsky and F. Y. Khalili, "Nonlinear meter for the gravitational wave antenna," Physics Letters A, vol. 218, pp. 167-174, Feb. 1996. 